\title{
Performance Evaluation Index Establishent and Application of a Novel Hybrid Mechanism for Automobile Electro-coating Conveying
}

\author{
Lei Feng ${ }^{1, a}$, Guoqin Gao ${ }^{2, b}$ \\ ${ }^{1,2}$ China, Jiangsu Province, Zhenjiang City, Xuefu Road, 301, Jiangsu University \\ aLeifengjiangsuedu@foxmail.com, bgqgao@ujs.edu.cn
}

\begin{abstract}
Keywords: parallel mechanism; transmission angle; motion/force transmissibility; optimum design. Abstract. For a novel hybrid mechanism for automobile electro-coating conveying, a local transmission index is proposed to be used for the optimal design the geometric parameters of the conveying mechanism. By defining the transmission angle of the mechanism, the local transmission index of the mechanism is defined according to the relationship between the force transmission characteristics and the transmission angle. Then the parameters of the lifting mechanism of the novel hybrid mechanism are designed by combining the performance evaluation index with the mechanism characteristics. Finally, kinematic control experiment is carried out to verify the correctness and validity of the performance evaluation index and the optimal design. The experimental results show that the mechanism designed using the index always operates far from any singularity with excellent motion/force transmissibility.
\end{abstract}

\section{Introduction}

The existing conveying mechanisms for electro-coating of automobile bodies, such as the RoDip conveyor and the multifunctional shuttle, are cantilever beam structure, which the ability to bear heavy load and large load is poorer and the level of flexibility is not high. Therefore, a novel hybrid mechanism for automobile electro-coating conveying is developed based on the hybrid mechanism. For the proposed novel hybrid conveying mechanism, it is more relevant to the engineering application needs through establishes the movement/force transmission performance evaluation index from the aspect of mechanics behavior characteristics.

Parallel mechanism is the main body of hybrid mechanism, and the research of hybrid mechanism is mainly based on parallel mechanism. In the design of parallel mechanism, performance evaluation index is a very important content, which is the basis of the design and the realization of the goal. Over the past 20 years, the Jacobi matrix, the condition number and the dexterity are used as the performance evaluation and design index. The index is originally proposed in the field of series mechanism, and successfully applied in the design of series mechanism. These research results are directly applied to parallel mechanism in the early stage [1-3], and followed up to now. At the same time, Merlet [4], a famous international parallel manipulator, had investigated two main performance indices which are widely used in the field of parallel mechanism: local conditioning index (LCI) and global conditioning index (GCI) [5], and suspected that these two index performance evaluation and optimization design in the parallel mechanism with mixing (movement and rotation) degrees of freedom.

Unlike serial mechanism for flexible operation, such as excavators of four-bar linkage, parallel mechanism is better at transfer motion and force. From the perspective of the structure constitutes, parallel mechanism and four-bar linkage are both belong to the closed-loop mechanism; from the perspective of the kinematic, parallel mechanism has the characteristics of the single closed loop four-bar linkage more or less. Four-bar linkage has experienced decades of application in industry, and its design and synthesis theory is proved to be scientific and feasible. This paper inherits the design thought of four-bar linkage, apply the concept of motion/force transfer characteristics to evaluate and design a novel hybrid conveying mechanism for electro-coating of automobile bodies. 
The paper defines the transmission angle learnt from the classical transmission angle concept of four-bar linkage and the local transmission index based on the relationship of force transmission characteristics and transmission angle to reflect motion/force transfer performance of a single position. To make the mechanism has higher speed and motion/force transmission performance and avoid the singular configuration, the transmission angle is selected in a certain range. To verify the design scheme, this paper designes the practical mechanism parameters based on the performance evaluation index. Finally, the kinematic control experiment for actual conveying mechanism is conducted to verify its effectiveness.

\section{Establishment of Performance Evaluation Index}

The novel conveying mechanism for electro-coating of automobile bodies consists of two parts: translation mechanism and lifting-rotating mechanism. The prototype of the novel conveying mechanism is shown in Fig. 1. The function of each part is relatively independent. As the main body, the structure of lifting-rotating mechanism is more complex, and have a greater impact on the conveying performance. Meanwhile, the rotating mechanism and lifting mechanism are separately controlled by the lifting driving motor and the rotating driving motor. Therefore, in order to characterize the motion/force transfer performance of the mechanism, this paper focuses on the research of the lifting mechanism.

\section{A. Structure description}

The lifting mechanism has two branches, each branch contains one PRR chain, the intersection piont $\mathrm{P}$ positioned by a couple of driving sliders in the workspace. Unilateral side structure diagram of lifting mechanism shown in Fig. 2, establishes a fixed reference coordinate system $O$-xy, where $P D_{1}=R_{1}, P D_{2}=R_{2}$, the distance of two sliders is $R_{3}$.

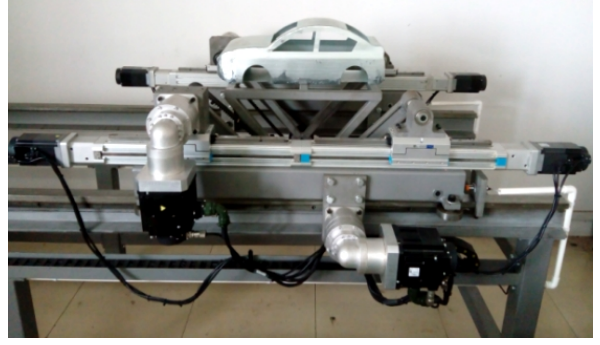

Fig. 1. The prototype of the novel conveying mechanism for electro-coating of automobile

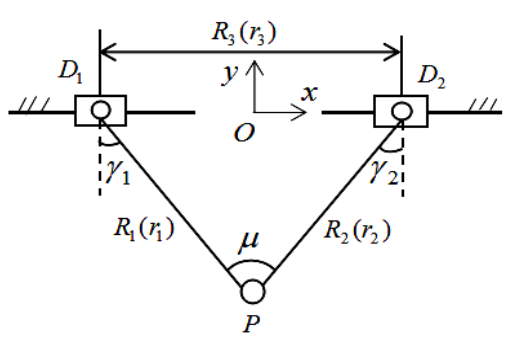

Fig. 2. Unilateral side structure diagram of lifting mechanism

The mechanism has three size parameters, namely $R_{1}, R_{2}$ and $R_{3}$, which values are from zero to infinity. To contain all mechanism which consists of different length of bars in a finite space, the physical properties of the mechanism should be eliminated. Defined

$D=\left(R_{1}+R_{2}+R_{3}\right) / 3$.

3 dimensional parameters $r_{i}(\mathrm{i}=1,2,3)$ can be obtained after processed:

$$
r_{1}=R_{1} / D, \quad r_{2}=R_{2} / D, \quad r_{3}=R_{3} / D \text {. }
$$

and there

$$
r_{1}+r_{2}+r_{3}=3 \text {. }
$$

To ensure that the lifting mechanism can be assembled and achieve two degrees of freedom, the $R_{1}$ and $R_{2}$ can not be 0 , and the $r_{1}+r_{2}$ can not be less than $R_{3}$, otherwise it will not be assembled into 
2-DOF parallel mechanism. Therefore, the scope of these 3 parameters should be

$$
0<r_{1}<3,0<r_{2}<3,0 \leq r_{3} \leq 1.5 \text {. }
$$

\section{B. Definition of performance evaluation index}

As shown in Fig. 1, $\gamma_{j}(j=1,2)$ is defined as the inverse transmission angle, $\mu$ is defined as the transmission angle. With two sliders as the drivers, when $D_{l} P$ reaches the horizontal position, that is $\gamma_{1}=90^{\circ}$, the force passed to the output point $\mathrm{P}$ from the first branch reaches the maximum. Similarly, when $\gamma_{2}=90^{\circ}$, the force passed to the output point $\mathrm{P}$ from the second branch reaches the maximum. We can see that the force transmission performance and transmission angle has a close relationship. The mechanism has the best force transmission performance when the transmission angle is $90^{\circ}$.

When $\gamma_{j}$ and $\mu$ is $0^{\circ}$ or $180^{\circ}$, the mechanism becomes singularities. It can be seen, the transmission angle also has a close relationship with the mechanism singular. In order to avoid the singular configurations and its nearby regions, $\gamma_{j}$ and $\mu$ can not be too small or too large. Through the above analysis, the transmission angle closer to $90^{\circ}$, the more preferable. To this end, defined an index of local transmission index as follows:

$$
\chi=\sin (T A) .
$$

where, $T A=\gamma_{j}$ or $T A=\mu$. In which

$$
0 \leq \chi \leq 1
$$

It is easy to see that the greater the value, the transmission angle closer to $90^{\circ}$, which means that the motion/force transfer performance of the mechanism is better. the index $\chi$ in this paper is the transmission performance evaluation index local transmission index.

In order to make the mechanism has a higher speed and motion/force transmission performance, the transmission angle is selected within the scope of $\left(45^{\circ}, 135^{\circ}\right)$. In this way, the limit value of local transmission index can be calculated accordingly. That is

$$
\chi>\sin (\pi / 4) .
$$

From the above analysis, it can be seen that in the limit of local transmission index, the transmission angles $\gamma_{j}$ and $\mu$ will not reach $0^{\circ}$ or $180^{\circ}$. It is also one of the significance of this paper that limiting the transmission angle can effectively avoid the singularity.

The minimum value of local transmission index can determine a workspace for the mechanism, the corresponding workingspace is good-transmission workspace. It is the set of all the pose of end effector reference point can be realized in the case of the local transmission index is greater than $\sin (\pi / 4)$.

\section{Design and Application}

As shown in Fig. 2, since the mechanism has only one active output - the output of the movement in the $\mathrm{y}$ direction. So there is no displacement in $\mathrm{x}$-direction.

The mechanism parameters that need to optimizal design are the length of two connecting rod $R$ $=R_{1}=R_{2}$ and the distence of two sliders in the zero position. According to the Eq. 6 can be known:

$$
\pi / 4<\gamma_{i}<\pi / 2, \pi / 2<\mu<3 \pi / 4 .
$$


where, $\gamma_{1}=\gamma_{2}$.

The the length of two connecting rod is designed as $R=R_{1}=R_{2}=500 \mathrm{~mm}$. The GTW value of the lifting mechanism along the $y$-axis can be calculated by the formula (16):

$$
\begin{aligned}
W_{y-G T W} & =R \cos \gamma_{\min }-R \cos \gamma_{\max } \\
& =500 * \cos \pi / 4-500 * \cos 3 \pi / 8 \\
& =162.2 \mathrm{~mm} .
\end{aligned}
$$

Expected working space along the $\mathrm{Y}$ axis direction of the length of the $100 \mathrm{~mm}$. That is, the end of the two connecting rod do sinusoidal motion with the amplitude of $50 \mathrm{~mm}$. So set the distance of two sliders in the zero position $R_{3}=800 \mathrm{~mm}$.

\section{Experimential Verification}

In the novel conveying mechanism for electro-coating of automobile bodies, the lifting mechanism is installed according to the size parameters optimizal designed by performance evaluation index. The actual length of the connecting $\operatorname{rod} R_{1}=R_{2}=495 \mathrm{~mm}$, and the actual distance of two sliders in zero position $R_{3}=800 \mathrm{~mm}$. Control the lifting mechanism to run the complete kinematics procedures, obtained the end trajectory of the connecting rod is shown in Fig. 3.

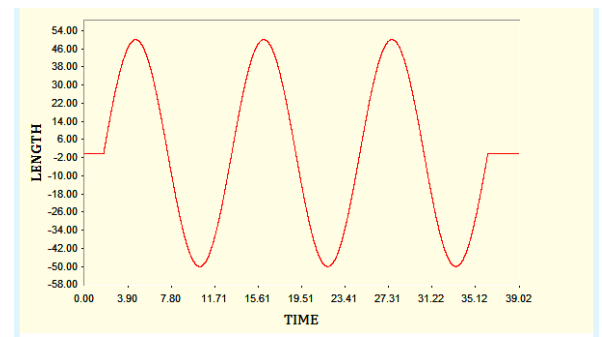

Fig. 3. The end trajectory of single side connecting rod of the lifting mechanism

When the mechanism rose to the highest point, the actual transmission angle: $\gamma_{1}=\gamma_{2}=60^{\circ} 47^{\prime}, \mu=121^{\circ} 34^{\prime}$.

When the mechanism fell to the lowest point, the actual transmission angle: $\gamma_{1}=\gamma_{2}=60^{\circ} 47^{\prime}, \mu=121^{\circ} 34^{\prime}$. The transmission angles were always satified with the local performance evaluation index in the process of operation and on singularities. The whole menchanism running smoothly, and had good motion/force transfer performance.

\section{Conclusion}

For a novel hybrid mechanism for automobile electro-coating conveying, to meet the requirements of the project application, this paper uses the concept of transmission angle to establishe a kind of parallel mechanism motion/force transmission performance evaluation index from the mechanical behavior characteristic angle. Then optimizal designs the lifting mechanism parameters with the performance evaluation index. Finally, through the experimental verification, the mechanism has good motion/force transmission performance and no singularities in the desired workspace.

\section{Acknowledgements}

This work is financially supported by National Natural Science Foundation of China (Grant No. 51375210), the Priority Academic Program Development of Jiangsu Higher Education Institutions, Zhenjiang Municipal Industry Key Technology R\&D Program (Grant No. GY2013062), the Science and Technology Project of Jingkou District of Zhenjiang City (Grant No. jkGY2013002) and Natural 
Science Foundation for Colleges and Universities in Jiangsu Province (Grant No. 15KJB510006).

\section{References}

[1] Chablat D and Wenger Ph. IEEE Transactions on Robotics and Automation. vol. 19 (2003), p. 403-410.

[2] Ottaviano E and Ceccarelli M. Robotica. vol. 20 (2002), p. 159-166.

[3] Carretero J A, Nahon M A and Podhorodeski R P. Int Journal of Robotics and Automation. vol. 15 (2000), p. 178-188.

[4] Merlet J P. Journal of Mechanical Design. vol. 128 (2006), p. 199-206.

[5] Gosselin C and Angeles J. ASME Journal of Mechanical Design. vol.113 (1991), p. 220-226. 